\title{
Type Synthesis Based on Modular Combination with Virtual Rotation Center and Application
}

\author{
Xinning Li $\mathbb{D}^{1},{ }^{1}$ Qishuo Zhang, ${ }^{1}$ Zongsu Zhang, ${ }^{1}$ Xianhai Yang $\mathbb{D}^{1},{ }^{1} \mathrm{Hu}$ Wu ${ }^{\mathbb{D}},{ }^{1}$ Yongbo Li, \\ and Hailong $\mathbf{Q u}{ }^{2}$ \\ ${ }^{1}$ School of Mechanical Engineering, Shandong University of Technology, Zibo 255000, China \\ ${ }^{2}$ Laizhou Jufeng Machinery Limited Company, Yantai 261400, China \\ Correspondence should be addressed to Xianhai Yang; yxh@sdut.edu.cn
}

Received 7 December 2021; Revised 11 February 2022; Accepted 15 February 2022; Published 3 March 2022

Academic Editor: Cheng Xu

Copyright (c) 2022 Xinning Li et al. This is an open access article distributed under the Creative Commons Attribution License, which permits unrestricted use, distribution, and reproduction in any medium, provided the original work is properly cited.

\begin{abstract}
Type synthesis of mechanical structure is of great significance to the realization of mechanism target function, systematization, and stability of mechanical device. The type synthesis method of multilinkage robot has been given high demands with increasing number of degrees of freedom and high flexibility in special occasions. In order to improve the workspace and flexibility of mechanism, this paper studies the existing type synthesis theory and proposes a type synthesis method of modular combination with virtual rotation centers. Firstly, modular units are built. Secondly, modular units are expanded according to the needed paths. In the end, the expanded modular units are combined to form the kinematic linkages. Based on the proposed method, the configuration design of the aerial working platform and the self-adaptive levelling platform is completed. The stabilities of two platforms are checked by modal analysis. The prototype products are manufactured, respectively, for further verifying validity of the method. The designed aerial working platform with virtual rotation centers can achieve $360^{\circ}$ rotating large workspace, more compact mechanical structure, and short arrival time at the same height than the common scissor-type and mast-type aerial working platforms. The designed adaptive levelling platform is tested that ensures the levelling of the upper surface at different inclinations. The method can provide new idea for the mechanism configuration and expand the application scope of new mechanisms.
\end{abstract}

\section{Introduction}

The transmission and transformation of mechanical motion is the basis of realizing the basic function of mechanical system. In the whole mechanical system, mechanism is the core of mechanical system. Mechanism innovation is the core of mechanical system innovation. The research of mechanism synthesis is the primary problem in modern mechanical design [1-4]. Multilinkage robot is a mechanism consisting of several moving pairs linked together by rigid rods. It is widely used in chemical, textile, metallurgy, petroleum, medical, and other fields with high flexibility, good obstacle avoidance performance, better singularity avoidance of mechanism, and easy to manufacture.
In the type synthesis design of the mechanism, Jia et al. [5] presented a general synthesis method for metamorphic mechanisms with scissor-like linkage, which offered more candidates for designers fabricated metamorphic mechanisms with deployment and grasping mobility. A new type of grasping submechanisms [6] was designed based on parallel mechanisms and metamorphic mechanisms. The work generated new ideas for synthesizing both single-DOF (degree of freedom) metamorphic mechanisms and 2-DOF parallel mechanisms. Wang et al. [7] presented an integrated method of knee rehabilitation and exoskeleton axis with automatic alignment. The correctness of motion compensation was verified by simulation, and the rationality of mechanism synthesis was confirmed. Zhang et al. [8] proposed a 
type synthesis method based on single-loop kinematic chain. Taking rotating pair and moving pair as design units, a kind of multimode moving mechanism was constructed. The feasibility and practicability of the configuration method were verified. Li et al. [9] proposed a simple and practical configuration method for 2R1T parallel mechanism based on Grassmann line geometry and mapping method. The synthesized mechanism reduced the singularity configuration, expanded the effective working space, and improved the motion performance of the mechanism. Lu et al. [10] studied the type synthesis of the 5-DOF parallel mechanisms with different submechanisms by utilizing digital topology graphs. Equivalent relations and merits were analysed between subplanar and spatial parallel mechanisms of 5-DOF parallel mechanisms. The complicated subparallel mechanisms were replaced with simple equivalent limbs. Li et al. [11] proposed a constructing approach for the 3-DOF single-loop parallel leg mechanism and designed a class of novel 3-DOF single-loop parallel leg mechanisms for walking robots. The novel leg mechanisms had larger step length and step height than the existing leg mechanisms. Zhang et al. [12] proposed a parallel mechanism type synthesis method with precisely constrained branches and obtained a variety of high-performance parallel mechanisms. Meng et al. [13] proposed a type synthesis method for parallel limbs and verified the method through a variety of configuration examples. Li et al. [14] proposed a type synthesis method based on Lie group theory for 3T1R parallel mechanism and simulated two specific 3T1R mechanisms. Gao et al. [15] studied the type synthesis method for the underactuated parallel mechanism in spherical motion mode. Sun and Huo [16] proposed a configuration method based on finite helix theory to solve the problem of motion restriction taking $1 T 2 \mathrm{R}$ parallel mechanism with accompanying motion as an example. Song et al. [17] proposed a synthesis method for 1T2R parallel mechanism based on the conformal geometric algebra theory and obtained configuration layout of the limbs and joints of the robots. Xie et al. [18] designed the configuration of 2T1R parallel manufacturing robot based on motion and constraint theory. These studies focused on type synthesis of a certain type of single mechanism, whose generality needed to be further verified. The configuration methods are not applicable for some specific situations with cumbersome configuration process and poor adaptability.

In order to improve the workspace and the flexibility of mechanism, this paper studied the type synthesis theory. The main contributions of this work were summarized as follows:

(1) The type synthesis method of modular combination with virtual rotation center was proposed. Based on the proposed method, the configuration design of the aerial working platform and the self-adaptive levelling platform was completed

(2) The aerial working platform was modally analysed and manufactured. It was verified that the designed aerial working platform with virtual rotation center achieved $360^{\circ}$ rotating large workspace, more compact mechanical structure, and shortened arrival time at the same height than common scissor-type and mast-type aerial working platform

(3) The designed adaptive levelling platform was modally analysed and manufactured. It was tested that the platform could ensure the levelling of the upper surface at different inclinations

The rest of this work was organized as follows. The proposed type synthesis method based on the modular combination with virtual rotation center was introduced in Section 2. The type synthesis, modal analysis, and performance verification of designed aerial working platform based on the proposed method were described in detail in Section 3. The type synthesis, modal analysis, and performance verification of designed self-adaptive levelling platform based on the proposed method were described in detail in Section 4. Finally, the research was summarized in Section 5.

\section{Type Synthesis Based on Modular Combination with Virtual Rotation Center}

2.1. Mechanism with Virtual Rotation Center. Rotation, as one of the basic motion forms, plays an indispensable role. Rotational components are connected to other components by rotation pairs as shown in Figure 1(a). However, there is a kind of mechanism has no actual rotation pair connecting rotational components as shown in Figures 1(b) and $1(c)$. The rotation center without actual rotation pairs is defined as the virtual rotation center (VRC). The mechanism with virtual rotation center is called VRC mechanism.

Remote center of motion (RCM) [19-24] and remote center of compliance (RCC) $[25,26]$ have virtual motion centers and the basic characteristics, whether the virtual center is near or not and the component is rigid or not. The mechanism with virtual rotation center has the following advantages compared with traditional mechanism.

(1) The physical rotation center of mechanism is vacant, which makes complex mechanisms simple and lightweight. On the premise of realizing the design function, the rotation center is set as virtual rotation center

(2) Other inevitable motion except functional movement of the design caused by rotating rigid members is called parasitic motion shown in Figure 2, also known as concomitant motion, which is not expected in design. It is the condition to be satisfied in the design of nonparasitic motion mechanism whether there is a fixed rotation center or continuous rotation axis

The rigid bar $O A$ shown in Figure 2 is connected to the base plate by a rotation pair. When bar $O A$ rotates around point $O$, the other end $A$ does an arc with $l$ radius. Point $A$ does approximately linear motion along $x$ axis when rotation angle is small. Thus, the motion along $y$ axis is parasitic motion, and its displacement is as follows:

$$
d_{A}=l \cdot(1-\cos \alpha)
$$




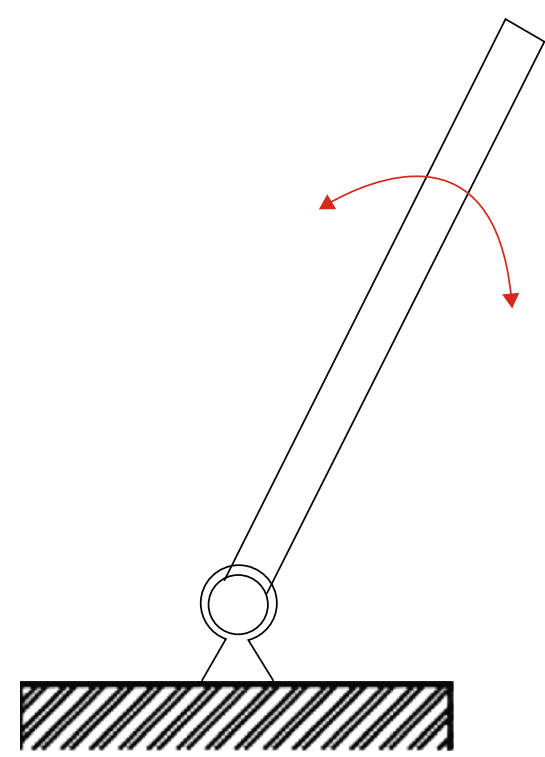

(a)

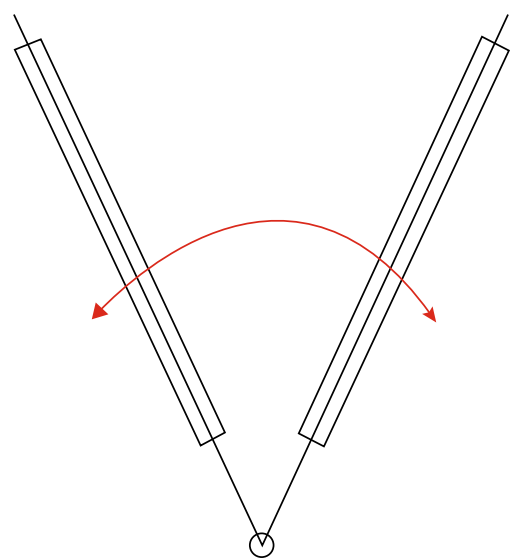

(b)

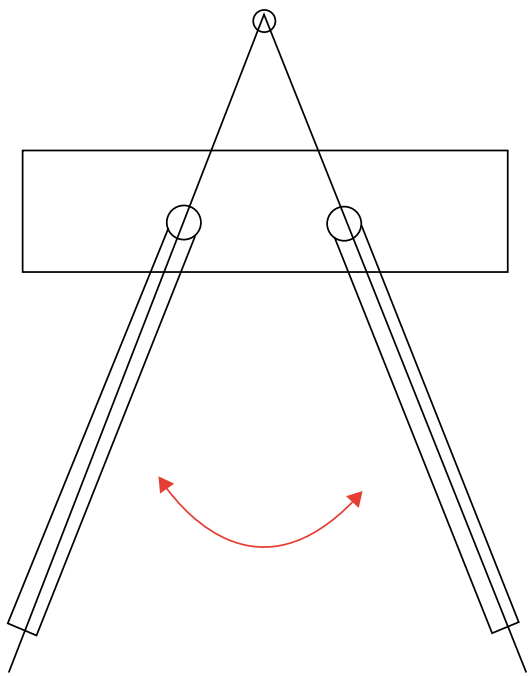

(c)

FIGURE 1: Rotation center and virtual rotation center.

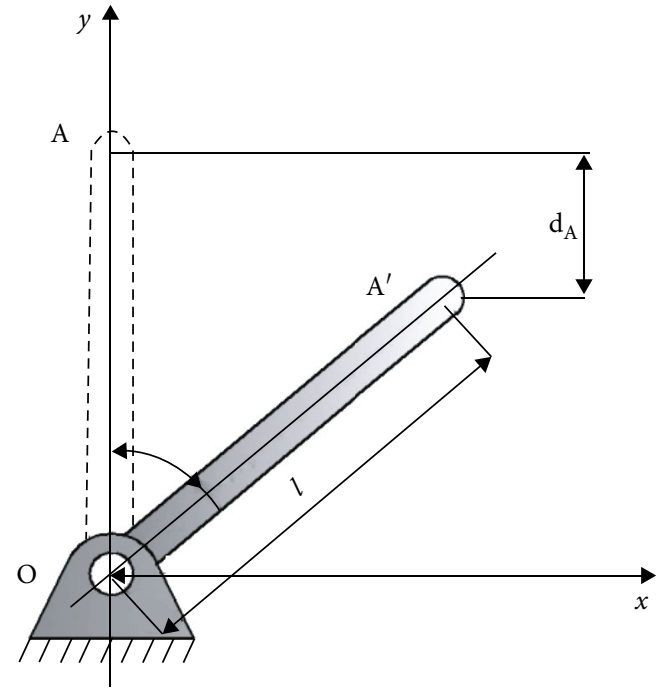

FIgURe 2: Parasitic motion of rigid link.

where $l$ is the length of bar $O A$ and $\alpha$ is rotation angle. Equation (1) after Taylor expansion is rewritten as follows:

$$
\begin{gathered}
d_{A}=l \cdot\left(1-\sum_{n=0}^{\infty} \frac{(-1)^{n}}{(2 n) !} \alpha^{2 n}\right) \\
=\frac{l}{2} \alpha^{2}-\frac{l}{24} \alpha^{4}+\frac{l}{720} \alpha^{6}-\cdots-\frac{(-1)^{n}}{(2 n) !} \alpha^{2 n}-o\left(\alpha^{2 n}\right) .
\end{gathered}
$$

Equation (2) can also be written as follows:

$$
d_{A}=a_{2} \alpha^{2}+a_{4} \alpha^{4}+a_{6} \alpha^{6}+\cdots+a_{k} \alpha^{k} .
$$

It can be concluded that the distance generated by the accompanying motion also increases rapidly with the increase of rotation angle when rotating linkages act as approximate linear motion. Parasitic motion can be removed with a relatively fixed rotation center.

(3) Compared with the fixed rotation center, the mechanism with virtual rotation center is beneficial to the design concept of modular regeneration, which can reduce the control difficulty and increase the reliability of the mechanism

(4) Mechanism with virtual rotation center can also improve the ability of mobile robot to leap over obstacles [27].

2.2. Type Synthesis Based on Modular Combination Theory. Type synthesis of mechanical structure is a qualitative form. The main task is to find possible solutions to resolve problems in the absence of exact algorithms, which can not only expand the scope of selection but also help to select a more appropriate or simple linkage.

At present, there are three theoretical systems for mechanism type synthesis research: the method based on screw theory, the displacement subgroup/submanifold method based on lie group lie algebra, and single-opened-chain method based on topology theory. Yang et al. [28] provided a systematic analysis and summary of the three methods. The relationship between three type synthesis methods is shown in Figure 3.

Module combination theory is widely used in mechanical engineering, chemical product synthesis, architectural design, and other fields. Module combination theory is used in type synthesis to find solutions and meet the design requirements.

2.2.1. Modular Unit Building. Modular units are divided into basic and combined types. The basic modular units include 


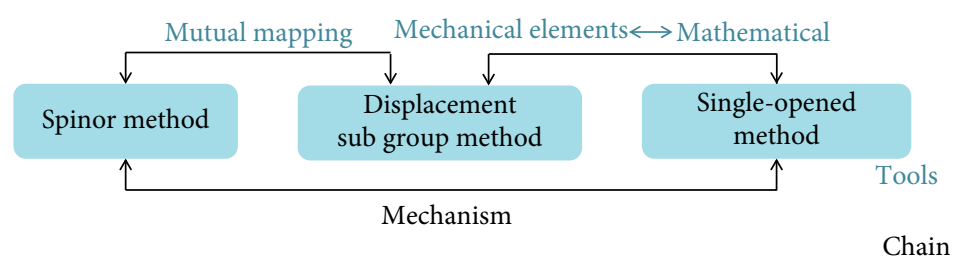

Figure 3: Relationship between three type synthesis methods.
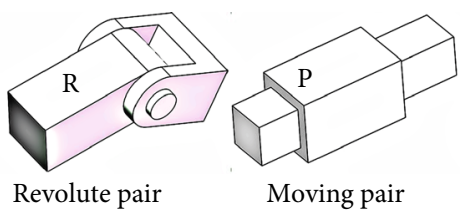

Moving pair

(a)

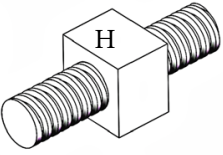

Screw pair

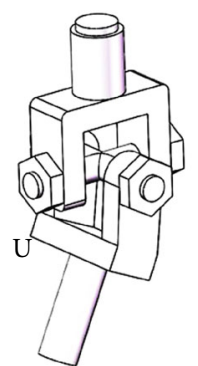

Ball stud pair

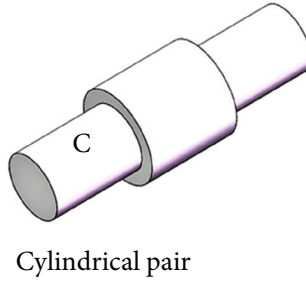

(b)

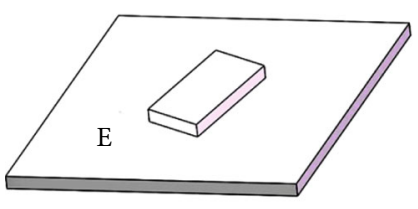

Plane pair

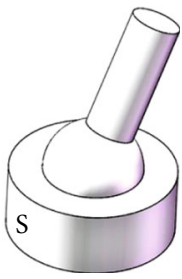

Ball pair

(c)

Figure 4: Basic modular units.

classes I, II, and III motion pairs. As shown in Figure 4(a), class I motion pairs include rotation pair, moving pair, and screw pair, which are represented by $\mathrm{R}, \mathrm{P}$, and $\mathrm{H}$, respectively. Class II motion pairs shown in Figure 4(b) include ball stud pair and cylindrical pair, which are represented by $\mathrm{U}$ and $\mathrm{C}$, respectively. Class III motion pairs shown in Figure 4(c) include plane pair and ball pair, which are represented by $\mathrm{E}$ and S, respectively. Most basic units are common modules, and combined module is composed of basic modular units.

2.2.2. Path Combination. Inspired by the mathematical model in the process of gene transcription and the application of multigene pathways in product synthesis, the modular units can be expanded according to the following paths:

\section{(a) Converse path}

Under the premise of meeting the required degree of freedom, the basic or combined modular units are directly converted to obtain the required motion chain.

\section{(b) Expand path}

Under the premise of meeting the required degree of freedom, the basic or combined modular units are directly expanded to obtain the required motion chain.

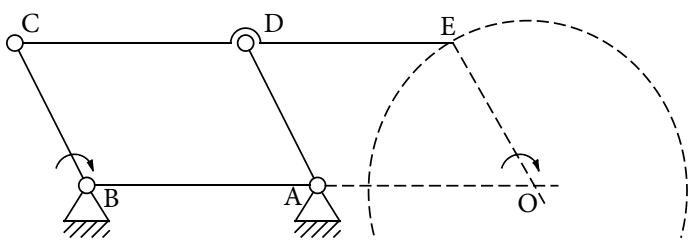

Figure 5: Parallel four-bar VRC mechanism.

(c) Connect path in series

Under the premise of meeting the required degree of freedom, the modular units are connected in series to obtain the required motion chain.

(d) Connect path in parallel

Under the premise of meeting the required degree of freedom, the modular units are connected in parallel to obtain the required motion chain.

(e) Connect path in series-parallel

Under the premise of meeting the required degree of freedom, the modular units are connected in series-parallel to obtain the required motion chain. 


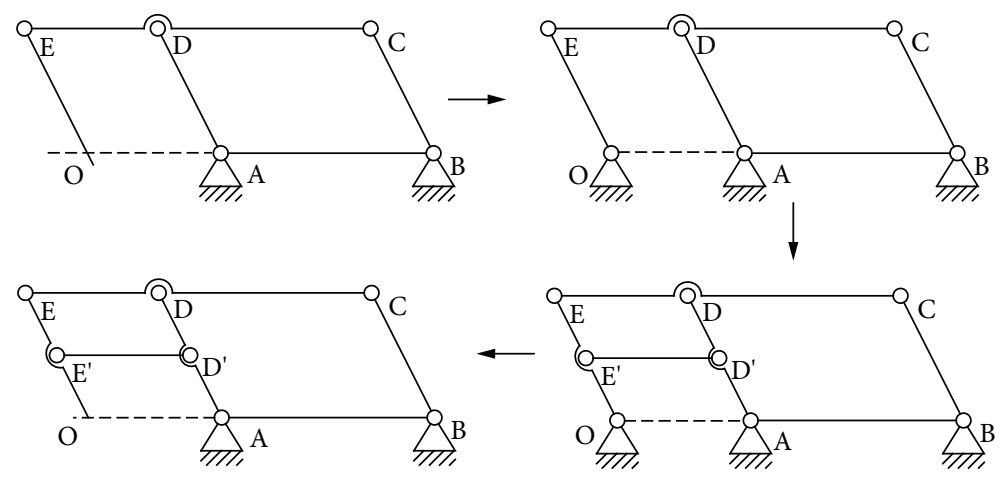

FIGURE 6: Expansion of VRC modular unit.

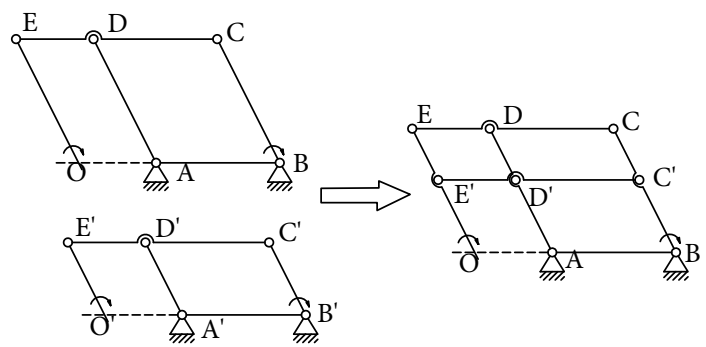

FIgURE 7: Combination of VRC modular units.

2.2.3. Modular Combination. Based on the dual rule between motion and constraint proposed by Blanding [28], the suitable path for specific design requirements is selected. Then, the modular units are combined to form a motion chain, and finally, the synthesis is completed.

It should be noted that the steps of modular unit building, path combination, and modular combination are not invariable. In the real configuration, the sequence may be changed, and several steps may be carried out simultaneously. For the selection of combination paths, one or several paths can be used at the same time.

\subsection{VRC Modular Unit and Type Synthesis}

2.3.1. VRC Modular Unit. The basis of VRC modular unit is parallel four-bar mechanism shown in Figure 5. The end actuator mechanism rotates around the virtual center. Point $E$ on the output component of the mechanism moves circularly around the fixed point $O$. There is no actual rotational pair at point $O$ actually, and point $E$ and point $O$ have only virtual constraints. Also, it can be seen that the components can be curved members according to the design requirements.

2.3.2. Expansion of VRC Modular Unit. The expansion type synthesis of single modular unit is shown in Figure 6. The rotation point $E$ of the VRC modular unit is connected with the virtual motion center (point $O$ ) by a linkage $E O$, and the two ends of the linkage are connected by rotation pair. Then, linkage $E^{\prime} D^{\prime}$ is put between $E O$ and $A D$. Finally, the rotation pair at point $O$ is removed to form a new VRC mechanism.

2.3.3. Combination of VRC Modular Units. The combination of VRC modular units is shown in Figure 7. The driving linkages $B C$ and $B^{\prime} C^{\prime}$ are collinear, the linkages $A D$ and $A$ ' $D$ ' are collinear, and the virtual center is point $O$, and then, two identical modular units are combined into a VRC mechanism. Of course, different modular units can also be combined into VRC mechanism.

2.3.4. Expansion of Multi-VRC Modular Units. Expansion of multi-VRC modular units is shown in Figure 8. Figure 8(a) is the basic configuration of parallel four-bar VRC mechanism. Four forms of VRC mechanisms shown in Figures 8(b)-8(e) can be obtained through modular expansion. If the position of the fixed end is changed, three forms of VRC mechanisms shown in Figures $8(\mathrm{f})-8(\mathrm{~h})$ can be obtained. In addition, it can adapt to the design requirements of different occasions that change the bending shape of the linkage and flexibly arrange the virtual motion center of the VRC mechanism.

\section{Type Synthesis of Aerial Working Platform}

Scholars have studied a series of devices for aerial working. The established aerial working platforms are divided into scissors lifting type [29, 30], mast type [31], telescopic arm type [32-34], and mechanical arm type [35-37]. The mechanical arm type among them has attracted more and more attention due to its wide workspace and strong operability. Combination with folding and telescopic [38], multiarm coordination $[31,39]$, or the combination with screw lifting mechanism [40] further improves the application of mechanical arm devices. However, the lifting and workspace of the manipulator device are directly related to the length and degree of freedom of the manipulator. The more the degree of freedom is, the more the manipulator 


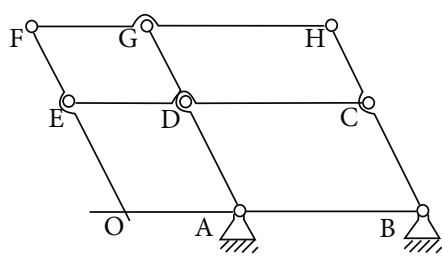

(a)

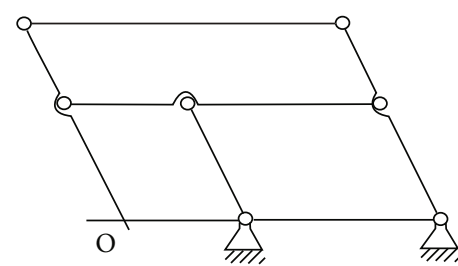

(c)

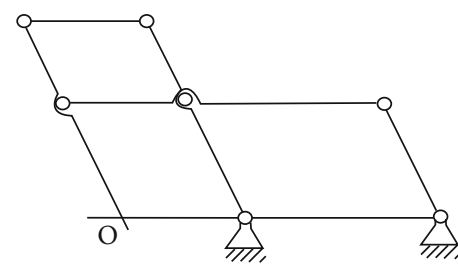

(e)

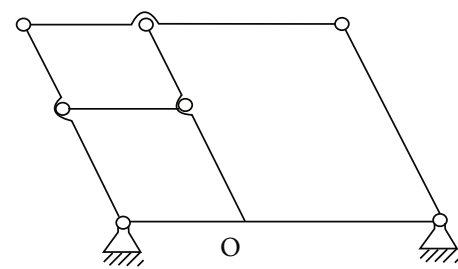

(g)

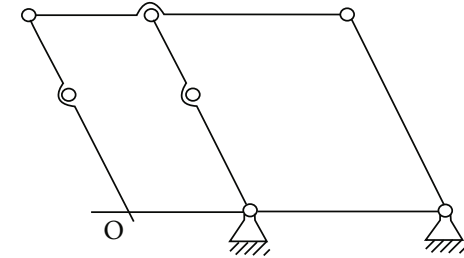

(b)

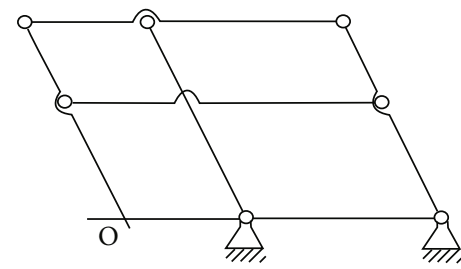

(d)

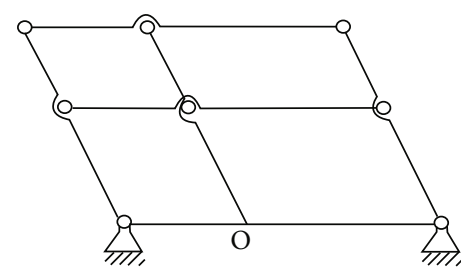

(f)

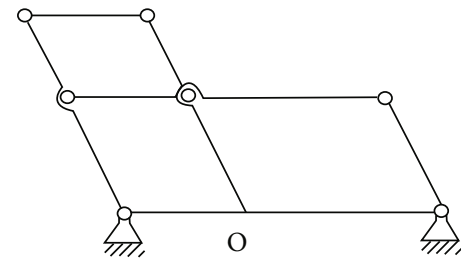

(h)

Figure 8: Expansion of multi-VRC modular units.

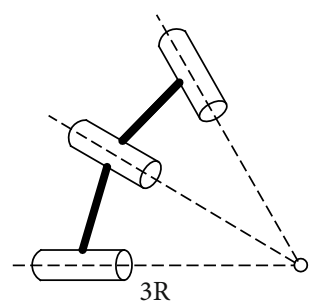

(a) Spherical sub-kinematic linkage units

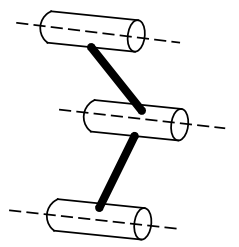

$3 R$
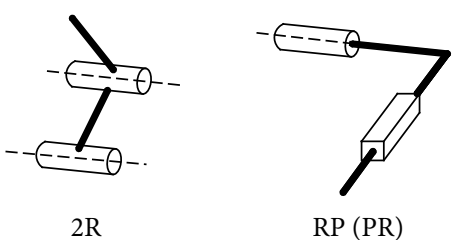

$\mathrm{RP}(\mathrm{PR})$

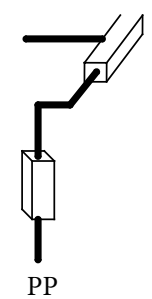

(b) Plane sub-kinematic linkage units

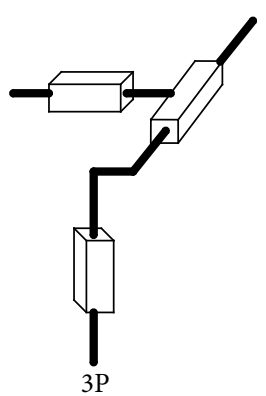

(c) Translational sub-kinematic linkage units 


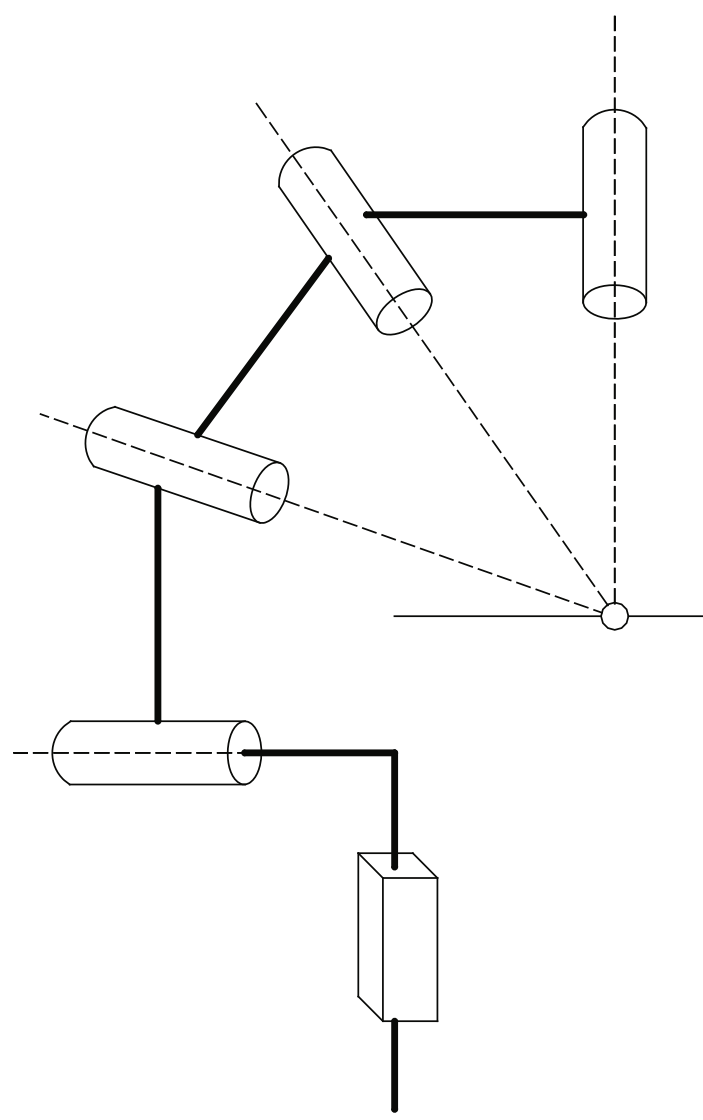

(a)

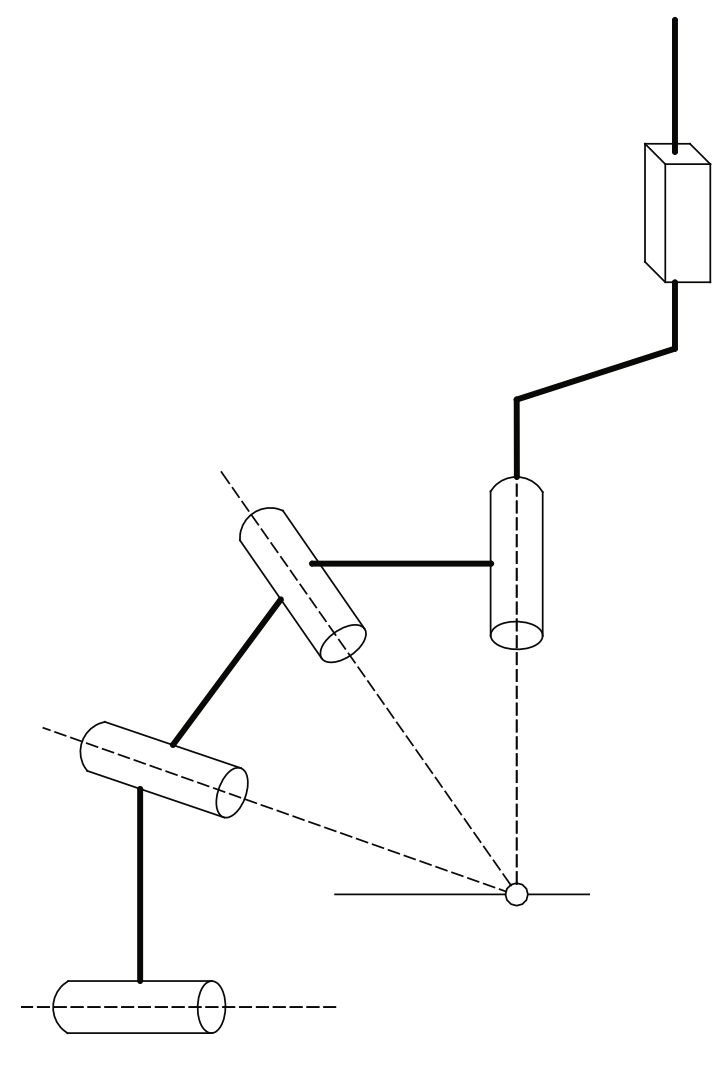

(b)

FIgURe 10: Kinematic units by moving kinematic pair.

configuration is, and the corresponding hardware cost will also increase. In order to improve the compactness and flexibility of the mechanism, expand the working space under the premise of minimizing the degree of freedom, and while ensuring load balance and smooth operation, the aerial working platform is designed as serial robotic arm based on the modular combination type synthesis with VRC.

\subsection{Type Synthesis of Series Robot}

3.1.1. Subkinematic Linkage Unit. It is the basis of linkage configuration that multiple kinematic pairs are combined by single-open chain to form subkinematic linkage module. The common subkinematic linkage units shown in Figure 9 include spherical subkinematic linkage units (Figure 9(a)), plane subkinematic linkage units (Figure 9(b)), and translational subkinematic linkage units (Figure 9(c)).

3.1.2. Combination of Kinematic Units. The kinematic units are combined to generate a new kinematic linkage based on the characteristics of moving pairs' free combination. As shown in Figure 10, the kinematic linkage shown in (b) is obtained by moving kinematic pair P in (a) to the kinematic linkage $(\mathrm{RRR})_{s}$.

Multiple kinematic linkages were obtained by combining the common kinematic pairs with different DOF, which is shown in Figure 11. (a) is RR(RRR) ${ }_{S}$ kinematic linkage. (b) is
$\mathrm{PR}(\mathrm{RRR}) \mathrm{S}$ kinematic linkage. (c) is $\mathrm{PP}(\mathrm{RRR})_{S}$ kinematic linkage. It can be seen that the kinematic linkages of various configurations can be obtained by modular combination of kinematic units.

3.1.3. Configuration under Multi-DOF. The common configurations of series robots under 4-DOF, 5-DOF, and 6-DOF are shown in Figure 12. The kinematic units contain the subkinematic linkage $(\mathrm{RRR})_{S}$.

3.2. Type Synthesis of Aerial Working Platform Based on the Series Robot. The VRC, which can make the mechanism high safety, simple structure, and lightweight, is introduced to ensure that the vertical direction of the passenger car remained unchanged. The configuration modular unit is constructed shown in Figure 13. (a) is the basis of the VRC mechanism. (b) is the parallelogram linkage mechanism to ensure the reliability of the passenger car. (c) is the basic unit simulation diagram of aerial working platform configuration.

In order to improve the working efficiency of aerial working platform in the lifting process and expand platform workspace, the aerial working platform serializes several basic or combined modular units to obtain the required kinematic linkage.

The rotation pair was added to the base part to consider the large-scale motion of the passenger car in threedimensional space. 


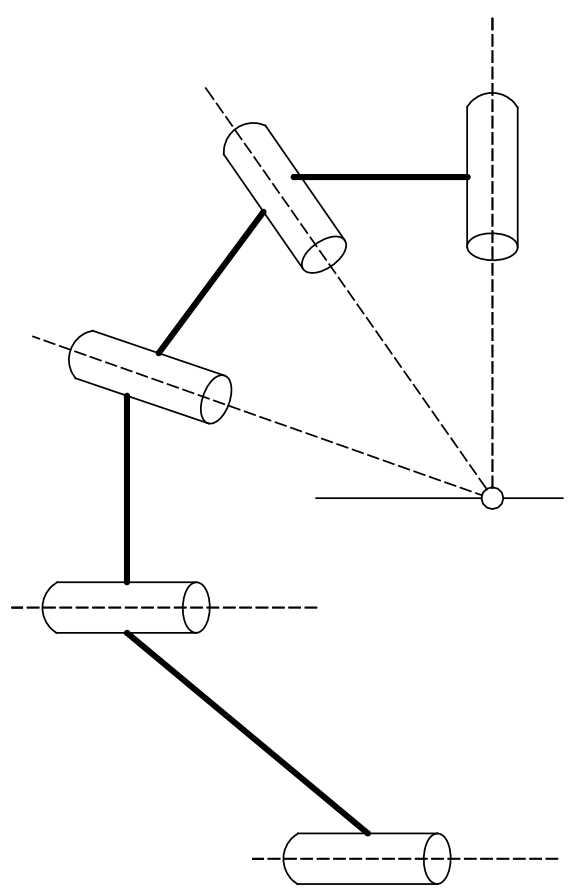

(a) $\mathrm{RR}(\mathrm{RRR})_{S}$

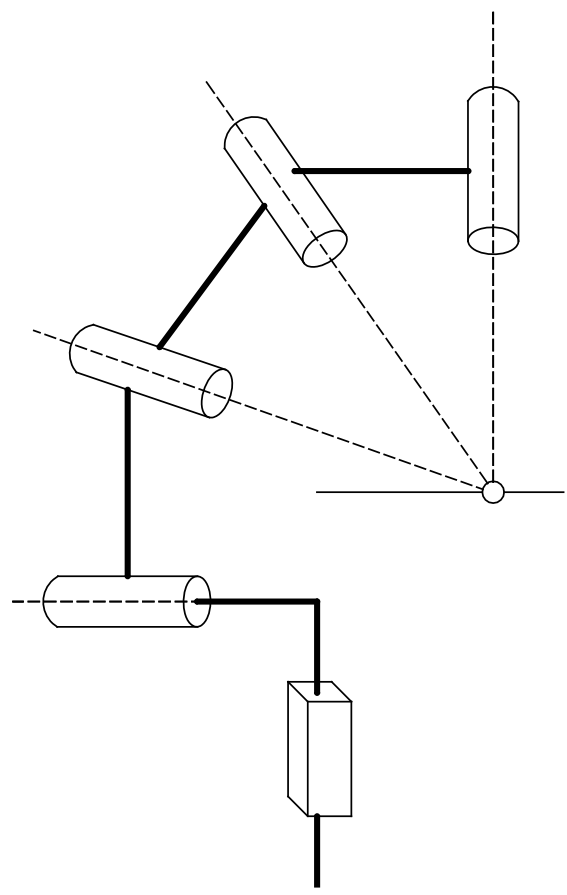

(b) $\mathrm{PR}(\mathrm{RRR})_{S}$

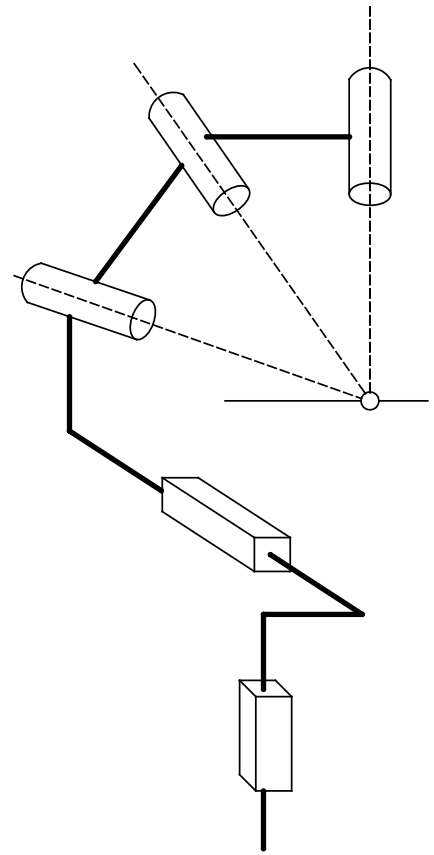

(c) $\mathrm{PP}(\mathrm{RRR})_{S}$

FIGURE 11: Kinematic units by combining with rotation pairs and moving pairs.
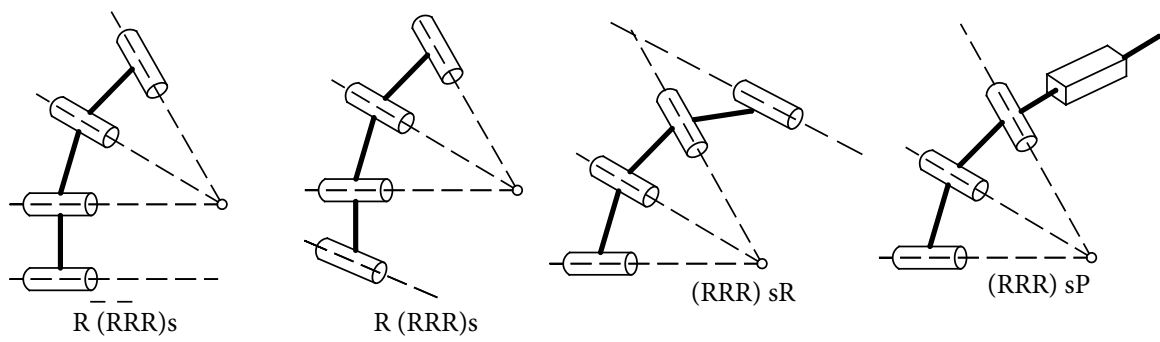

$4 \mathrm{DOF}$
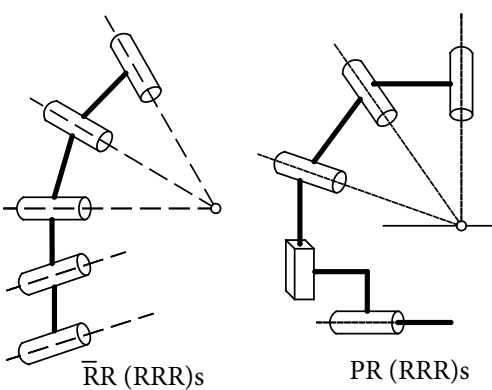

$5 \mathrm{DOF}$

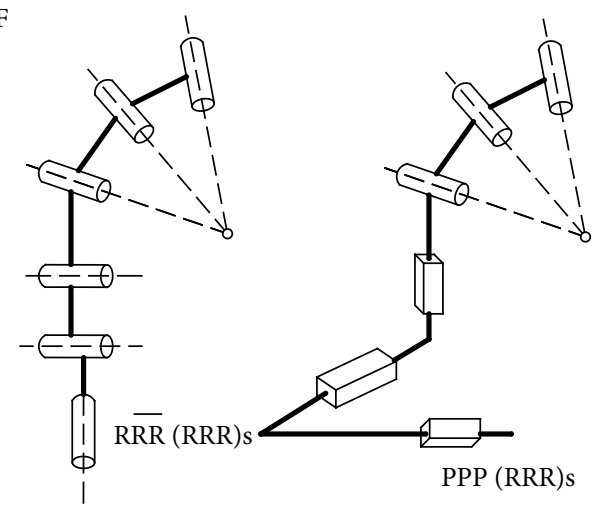

Figure 12: Configuration under multi-DOF.

Combining the modular unit shown in Figure 13 with 3R subkinematic linkage, the modular combination configuration of aerial working platform was built. The product prototype is shown in Figure 14. The designed aerial working platform adopted folding arm structure. The working device with $360^{\circ}$ full rotary was hydraulically driven. In order to realize the good stability and sufficient power in the opera- tion of applied engineering machinery, the linkage lifting mechanism adopted PLC to control hydraulic driving. The working arm was composed of three section folding arms, which were folded and closed in not-working condition and was extended by hydraulic device when working. The maximum working height of the platform is $7 \mathrm{~m}$, the maximum working radius is $3 \mathrm{~m}$, and the maximum load is 


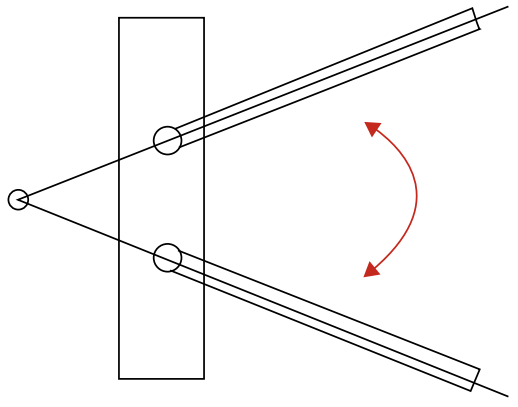

(a)

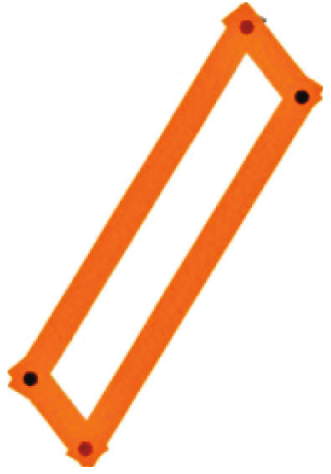

(b)

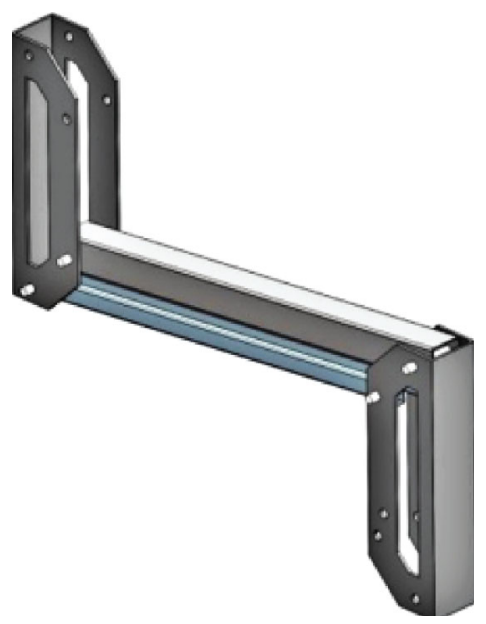

(c)

FIGURE 13: Configuration modular unit of aerial working platform.

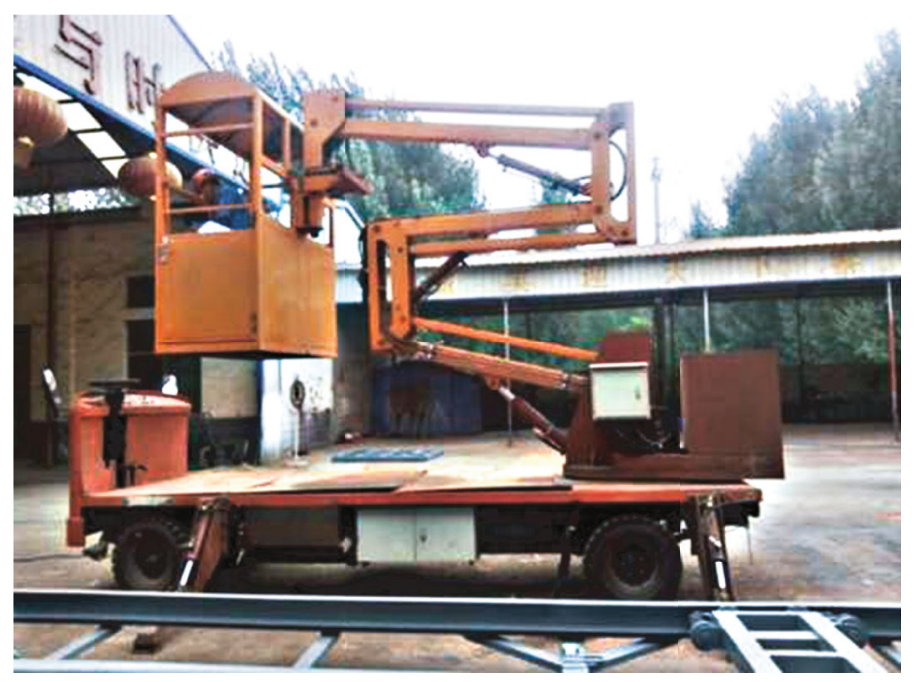

Figure 14: Prototype of aerial working platform. 

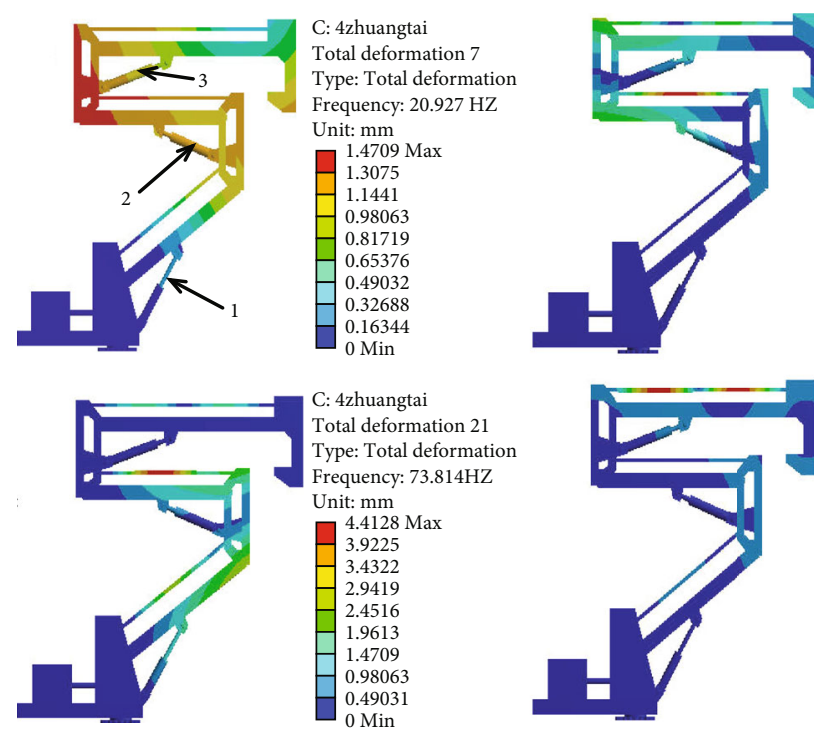

C: 4zhuangtai Total deformation 12 Type: Total deformation Frequency: $45.025 \mathrm{HZ}$ Unit: $\mathrm{mm}$ 4.0368 Max $-3.5883$ -3.1398
-2.6912
-2.2427
-1.7941
-1.3456
-0.89707
-0.44854
0
0 Min

C: 4zhuangtai Total deformation 26 Type: Total deformation Frequency: $97.054 \mathrm{HZ}$ Unit: $\mathrm{mm}$

\begin{aligned} $5.7056 \mathrm{Max} \\ -5.0717 \\ 4.4377 \\ 3.8037 \\ 3.1698 \\ 2.5358 \\ 1.9019 \\ 1.2679 \\$\hline 0.63396 \\ $0 \mathrm{Min}\end{aligned}$

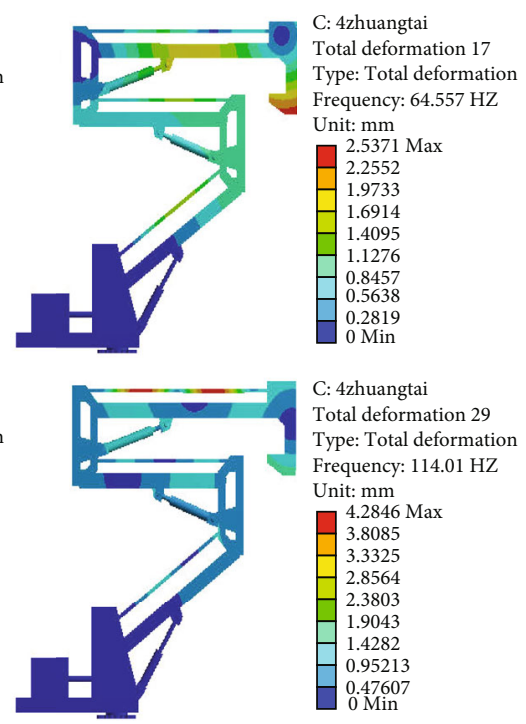

(a) Modal clouds of the No. 1 posture

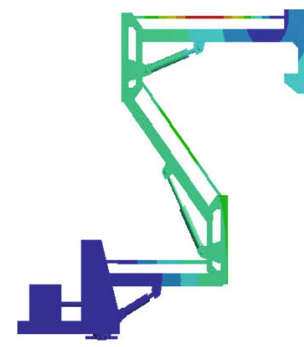

C: 4zhuangtai Total deformation 7 Type: Total deformation Frequency: $27.327 \mathrm{HZ}$ Unit: $\mathrm{mm}$

\begin{tabular}{|l}
$2.8453 \mathrm{Max}$ \\
2.5292 \\
2.213 \\
1.8969 \\
1.5807 \\
1.2646 \\
0.94844 \\
0.63229 \\
0.31615
\end{tabular}

C: 4zhuangtai Total deformation 23 Type: Total deformation

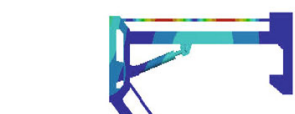

Frequency: $78.854 \mathrm{~Hz}$ Unit: $\mathrm{mm}$ - $\begin{array}{r}4.8443 \\ 4.3061 \\ 3.7678\end{array}$ 3.2295
-2.6913 2.6913 2.153
-1.6148
1.0765 1.0765
0.53826 0 Min

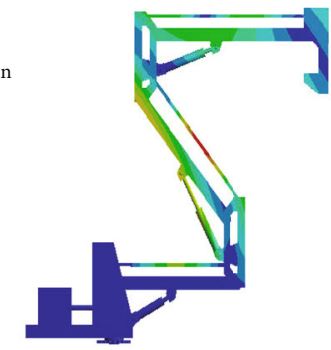

C: 4zhuangtai

Total deformation 14 Type: Total deformation Frequency: $51.056 \mathrm{HZ}$ Unit: $\mathrm{mm}$ $3.1385 \mathrm{Max}$ - 2.7898 2.441
-2.0923 1.7436 1.7436
-1.3949 $-1.0462$ 0.69744

0 Min

C: 4zhuangtai

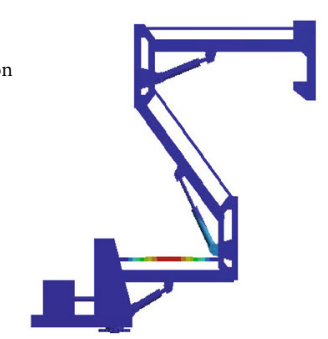

Total deformation 25 Type: Total deformation Frequency: $80.875 \mathrm{HZ}$ Unit: $\mathrm{mm}$

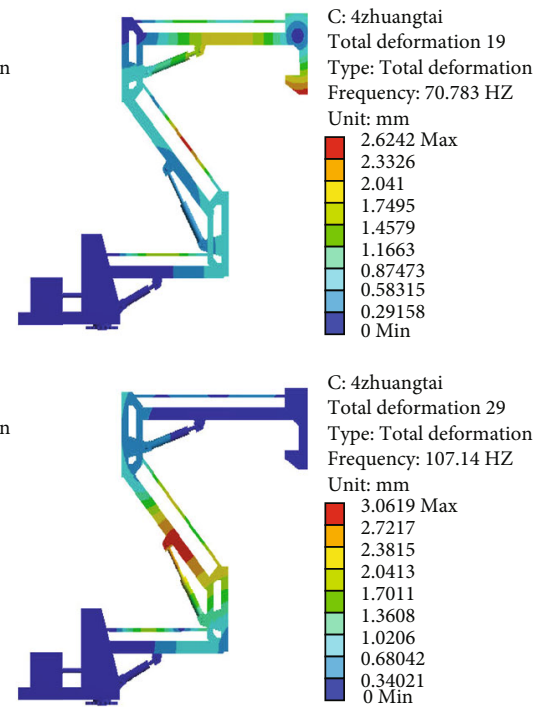

(b) Modal clouds of the No. 2 posture

Figure 15: Continued. 


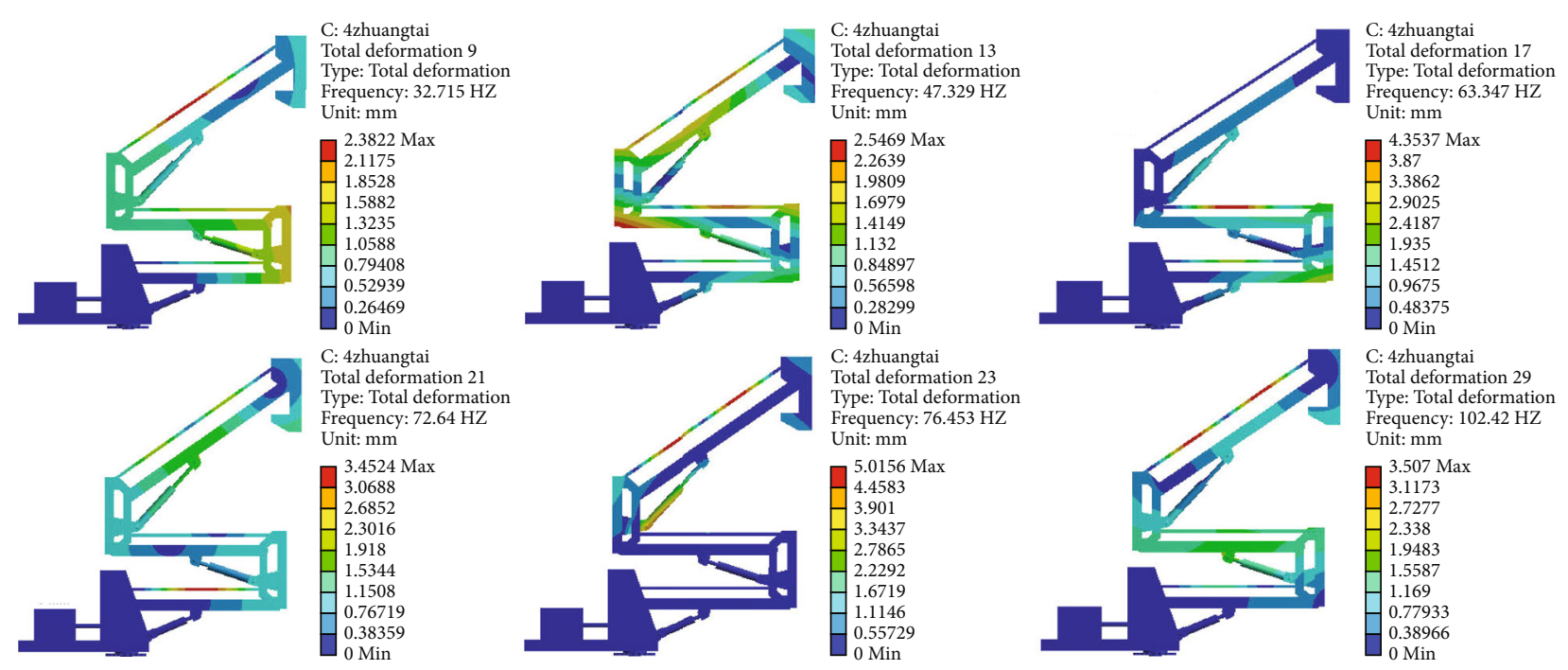

(c) Modal clouds of the No. 3 posture
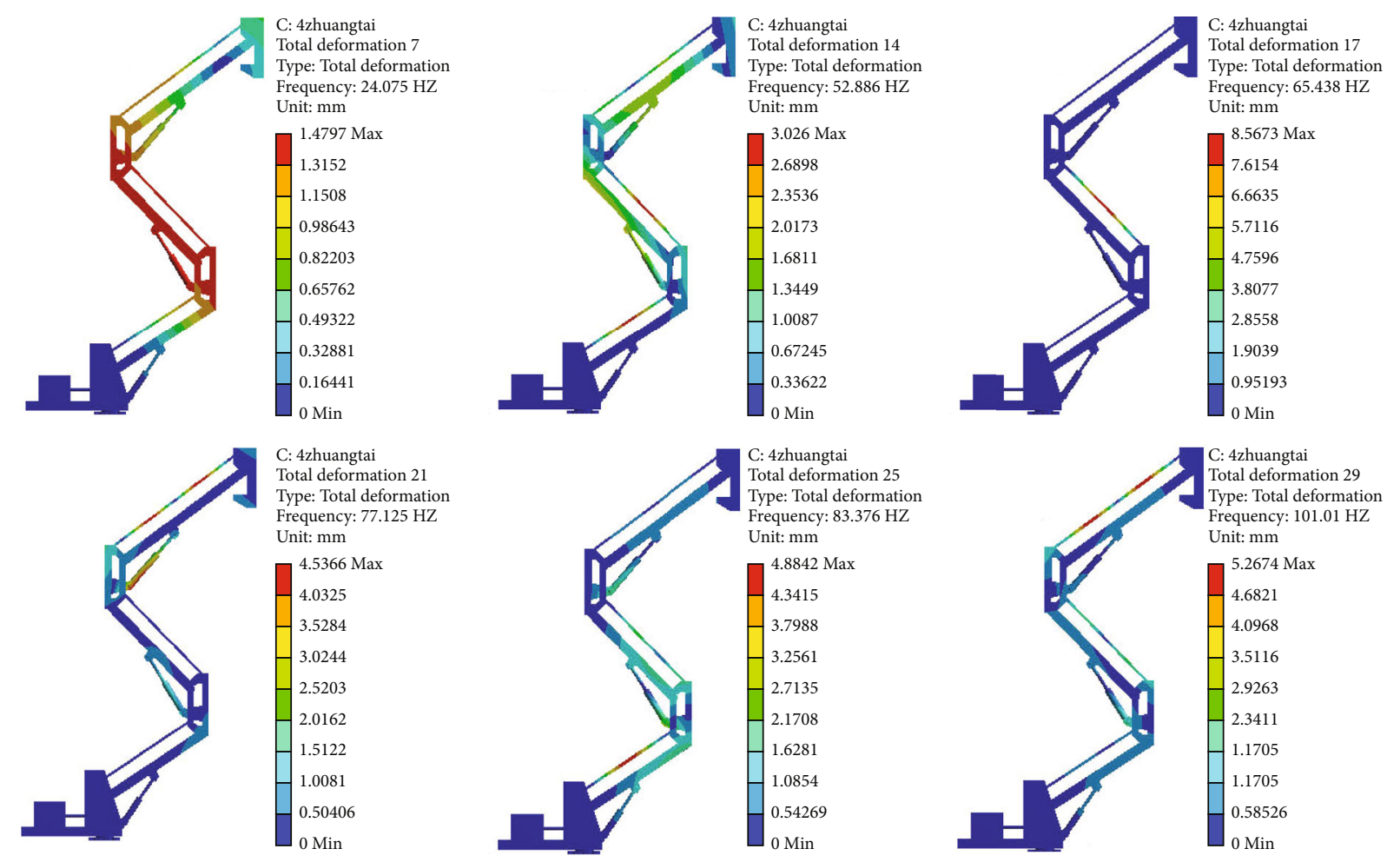

(d) Modal clouds of the No. 4 posture

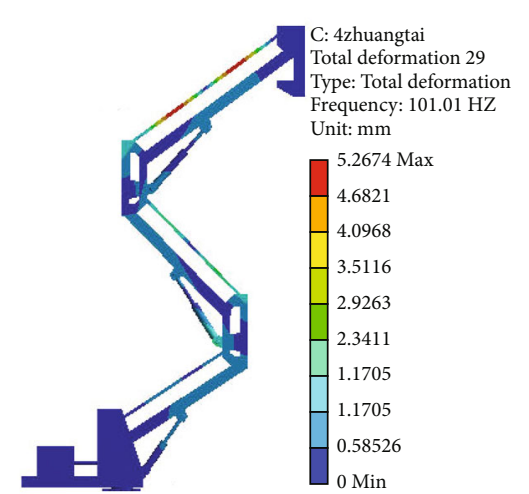

FIGURE 15: Vibration cloud figures of aerial working platform.

$1000 \mathrm{~kg}$, which can meet the space and height requirements of general aerial working.

3.3. Modal Analysis of Designed Aerial Working Platform. In order to verify the stability of the mechanism, the modal analysis of designed aerial working platform was carried out. The linkages material was set as structural steel. The hydraulic cylinder was thought filled with oil. ANSYS Workbench was used to conduct 30-order modal simulation analysis on four postures of aerial working platform. The first six orders were not analysed because of small frequencies, uni- form, and small deformations. The selected six modal clouds of representative natural frequency range for every one posture are shown in Figure 15. Three hydraulic cylinders were set to 1,2 , and 3 from bottom to top, respectively. As shown in (a), the piston of the No. 1 hydraulic cylinder protruded to the maximum position. The pistons of the No. 2 and No. 3 hydraulic cylinders had no any extension. The piston of the No. 2 hydraulic cylinder protruded to the maximum position, and the pistons of the No. 1 and No. 3 hydraulic cylinders had no any extension in (b). The piston of the No. 3 hydraulic cylinder protruded to the maximum 


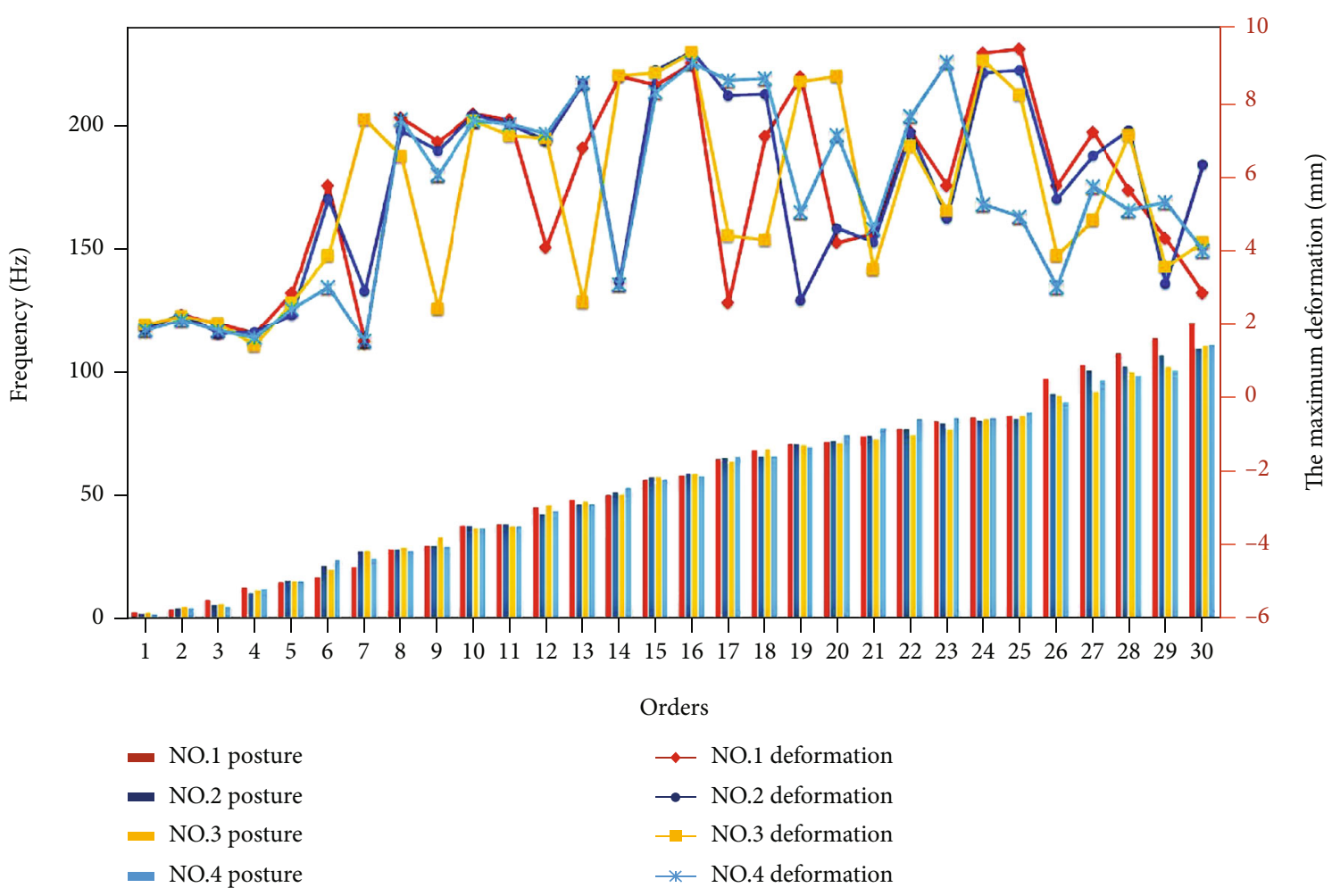

FIGURE 16: Frequency and deformation of aerial working platform at four postures.

position, and the pistons of the No. 1 and No. 2 hydraulic cylinders had no any extension in (c). All pistons of hydraulic cylinders expanded to the maximum position as shown in (d). It can be seen that the maximum vibration deformation of the mechanism is generally concentrated in the slender rods of the parallelogram structure. When the overall structure deformation is uniform, the maximum deformation is about $5 \mathrm{~mm}$, which will not affect the normal operation of the platform because there is no high precision requirement. When the deformation is concentrated on slender rod, as shown in (b5) and (d3), the deformation is close to $9 \mathrm{~mm}$, but the deformation of other linkages is close to $1 \mathrm{~mm}$, which have no impact on the working of the platform. The slender rod of the parallelogram structure buffers the vibration at the natural frequency. The product prototype, which met the requirements of engineering practice, verified the feasibility of the modular combination comprehensive theory.

The 30-order natural frequencies and corresponding deformation of the aerial working platform at four postures are shown in Figure 16. The bar charts based on principal coordinates axis represent the natural frequencies of four postures at different orders, respectively, and the broken line charts based on subordinate axis represent the maximum deformations of four postures at different orders, respectively. It can be seen that the natural frequencies of the four postures under each order are consistent and almost the same. The No. 1 posture obtained the lower deformations at $7,12,17,20$, and 30 orders than other three postures. The No. 2 posture obtained the lower deformations at 3, 5, 19, 23, and 29 orders than other three postures. The No. 3 posture obtained the lower deformations at $4,8,9,10,11,13,18,21$, and 27 orders
TABLE 1: Comparison of the time spent of aerial working platforms with the same height.

\begin{tabular}{lccc}
\hline Lifting height $(\mathrm{m})$ & Scissor type & $\begin{array}{c}\text { Time }(\mathrm{s}) \\
\text { Mast type }\end{array}$ & $\begin{array}{c}\text { Designed } \\
\text { configuration }\end{array}$ \\
\hline 4 & 8.52 & 6.83 & 5.16 \\
5 & 10.31 & 8.22 & 7.21 \\
6 & 12.06 & 10.75 & 9.31 \\
\hline
\end{tabular}

than other three postures. The No. 4 posture obtained the lower deformations at $1,2,6,14,15,16,22,24,25,26$, and 29 orders compared with the other three postures. It was obtained that that No. 3 and No. 4 postures generated the lower deformation, whose hydraulic cylinders at the top expanded to limit position. Combined with the previous model cloud images, the amplitude changes of corresponding deformations are basically consistent. It is verified that the configuration design of the whole structure is stable and feasible. Combined with corresponding components, the deformations meet the common working requirement of aerial working platform. It is not necessary to remove the natural frequencies from the operating frequencies.

3.4. Experimental Verification of Performance. In order to verify the performance of the designed aerial work platform based on the modular combination type synthesis with VRC, the time of designed platform taken from one height to another was compared with that of the scissor-type [30] and mast-type [41] aerial working platforms. The results are shown in Table 1. 


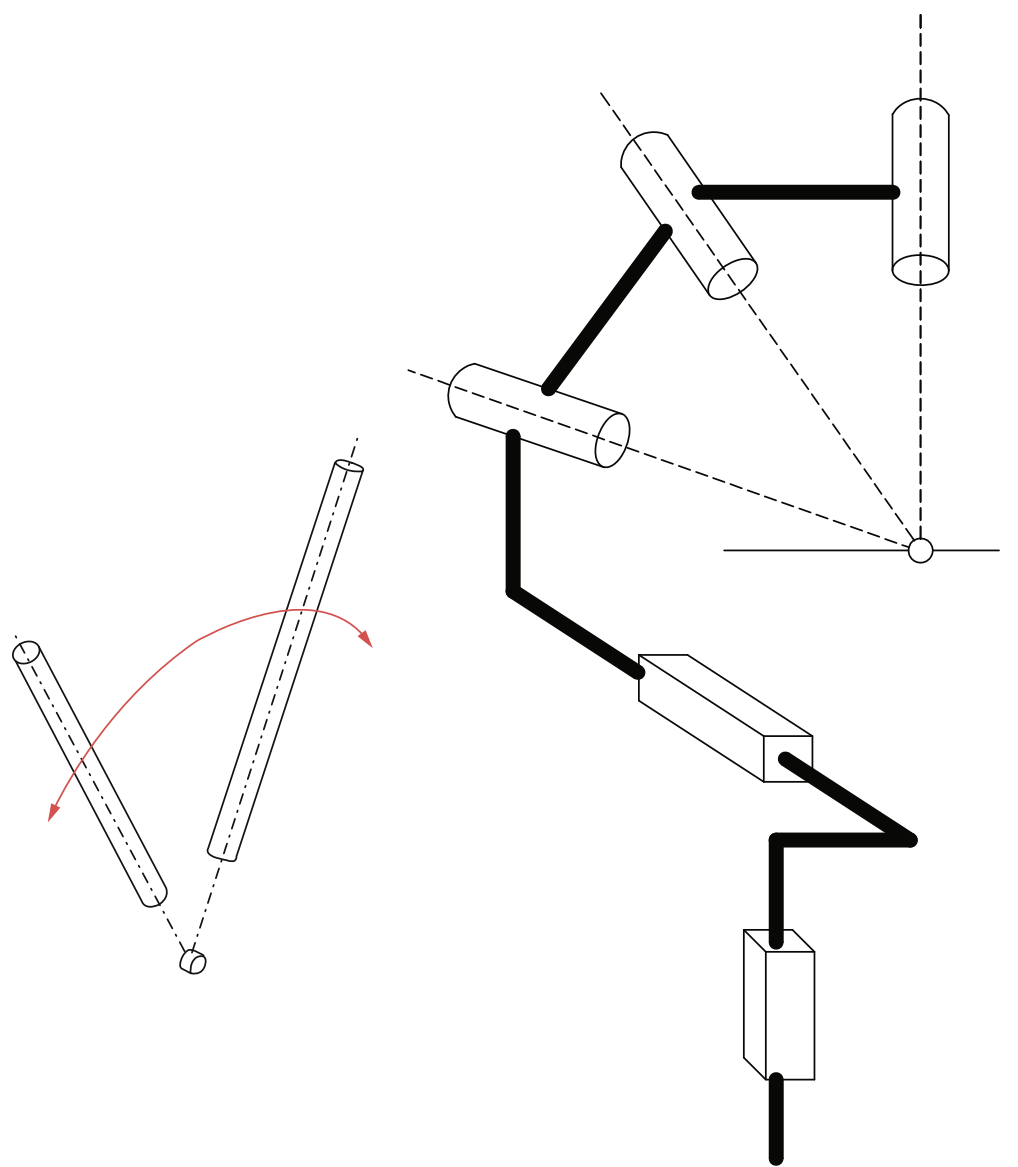

(a)

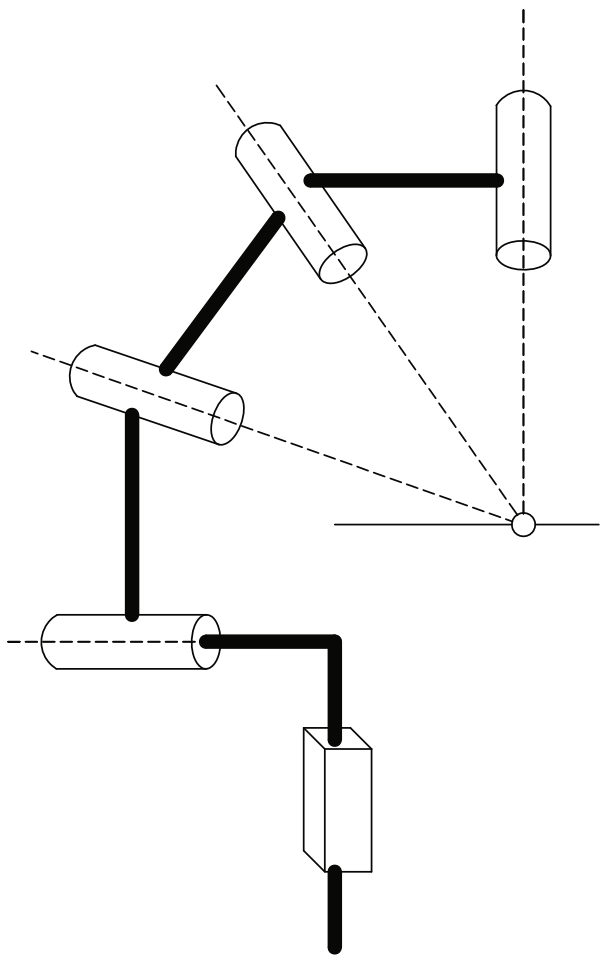

(c) (b)

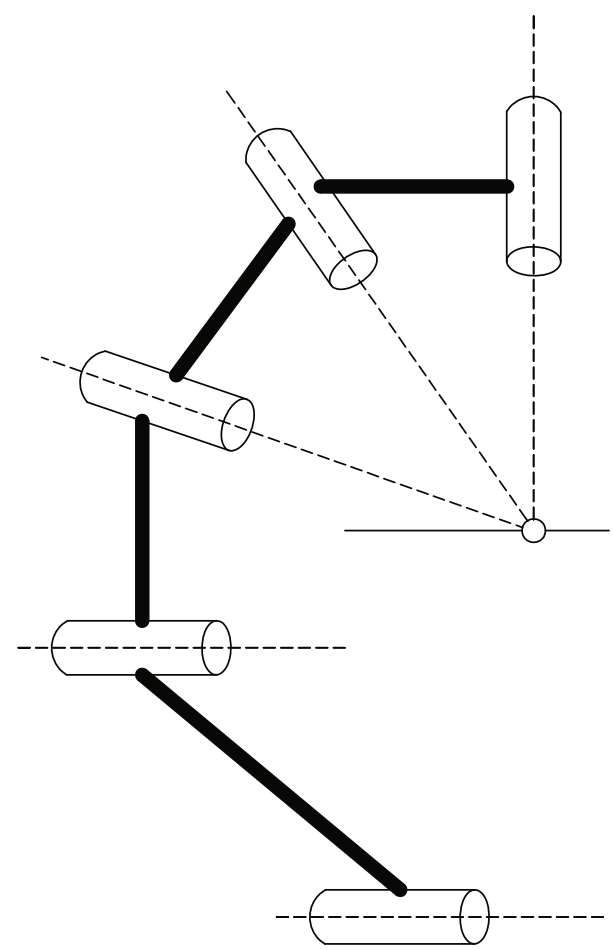

(d)

FIgUre 17: Modular units of adaptive levelling platform. 

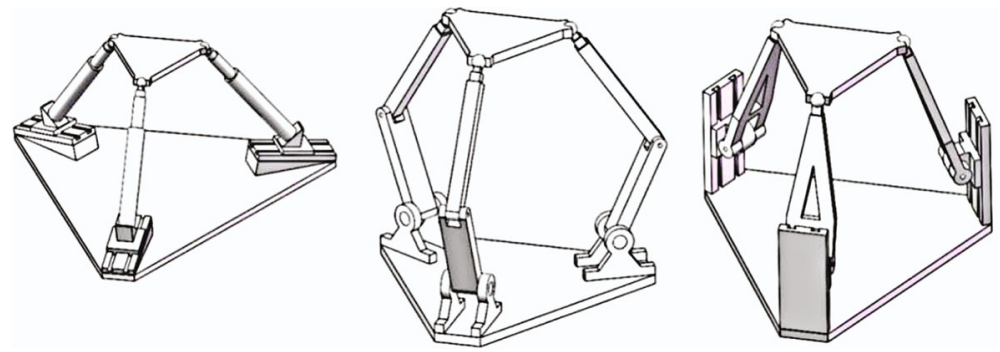

Figure 18: Combinations of modular units.

It can be seen that the spent time of the designed configuration scheme is the least. The aerial working platform designed based on the proposed type synthesis has higher operating efficiency, which is $30.77 \%$ shorter than that of scissor type and $16.97 \%$ shorter than that of mast type.

Compared to the $\mathrm{P}^{(4 \mathrm{R})}$-type synthesis method [42] based on topology theory, it can be seen from the process of configuration design that the type synthesis method based on modular combination theory avoids complicated formula derivation. The configuration design is complete by modular combination to generate kinematic linkage under the given function. The design process is relatively simple. It is easy for mechanical designers.

\section{Type Synthesis of Self-Adaptive Levelling Platform}

As the bottom component of aerial working platform, the stability of the base affects the stable operation of the upper linkage robot directly. In the case of unsatisfactory road conditions on the ground, the function of adaptive levelling platform is particularly critical. At present, the levelling system mainly has three supports, four supports, six supports, etc. The carrier carried by the platform plane is in a horizontal state by the adjustment of the mechanical mechanism. The design of mechanical mechanism determines the levelling performance directly. The mechanical structure needs to level the plane. The open linkage solution is difficult to achieve the purpose of levelling, so the parallel structure is taken to complete the levelling function based on the series kinematic units.

4.1. Type Synthesis of Self-Adaptive Levelling Platform. High flexibility is required for plane levelling, so the modular unit needs ball pairs. The selected modular units are shown in Figure 17. (a) is the mechanism module for VRC. (b-d) are the constructed subkinematic linkage modules. (b) is PPS type. (c) is PRS type. (d) is RRS type.

The platform is configured according the selected modular units. The several combinations of modular units are shown in Figure 18.

An equivalent ball pair was designed to meet levelling requirements and improve the activity range of levelling platform. As shown in Figure 19, the equivalent spherical pair consisted of linkages connected by two axes pins, whose ends were installed a pair of angular contact ball bearings.

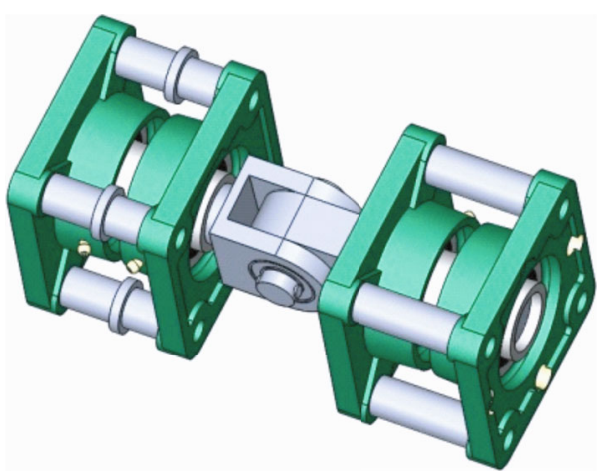

FIGURE 19: Equivalent ball pair.

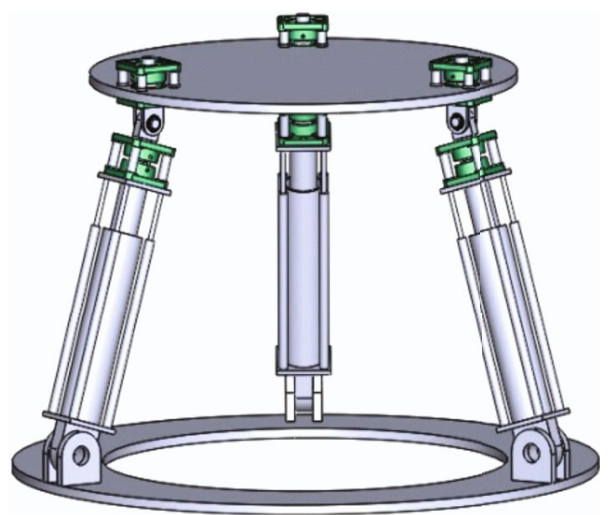

FIGURE 20: Configuration of the self-adaptive levelling platform.

From the angle of easy manufacturing and control, the scheme of Figure 17(c) PRS was selected. The PRS kinematic linkage was exchanged to form RPS kinematic linkage to realize levelling better. Configuration of the self-adaptive levelling platform is shown in Figure 20.

4.2. Modal Analysis of Designed Self-Adaptive Levelling Platform. Based on vibration principle, the 10-order modal analysis of the levelling platform was carried out to study its natural vibration characteristics. As shown in Figure 21, the 2th-10th order modal cloud diagrams were displayed in turn from left to right and from top to bottom. The 2th-6th order natural frequencies were concentrated in $48-123 \mathrm{~Hz}$. It can be seen that the vibration deformation is mainly concentrated at the upper platform in this range of natural frequencies. The 7-10-order natural frequencies are concentrated in $177-179 \mathrm{~Hz}$, and the mode shape 


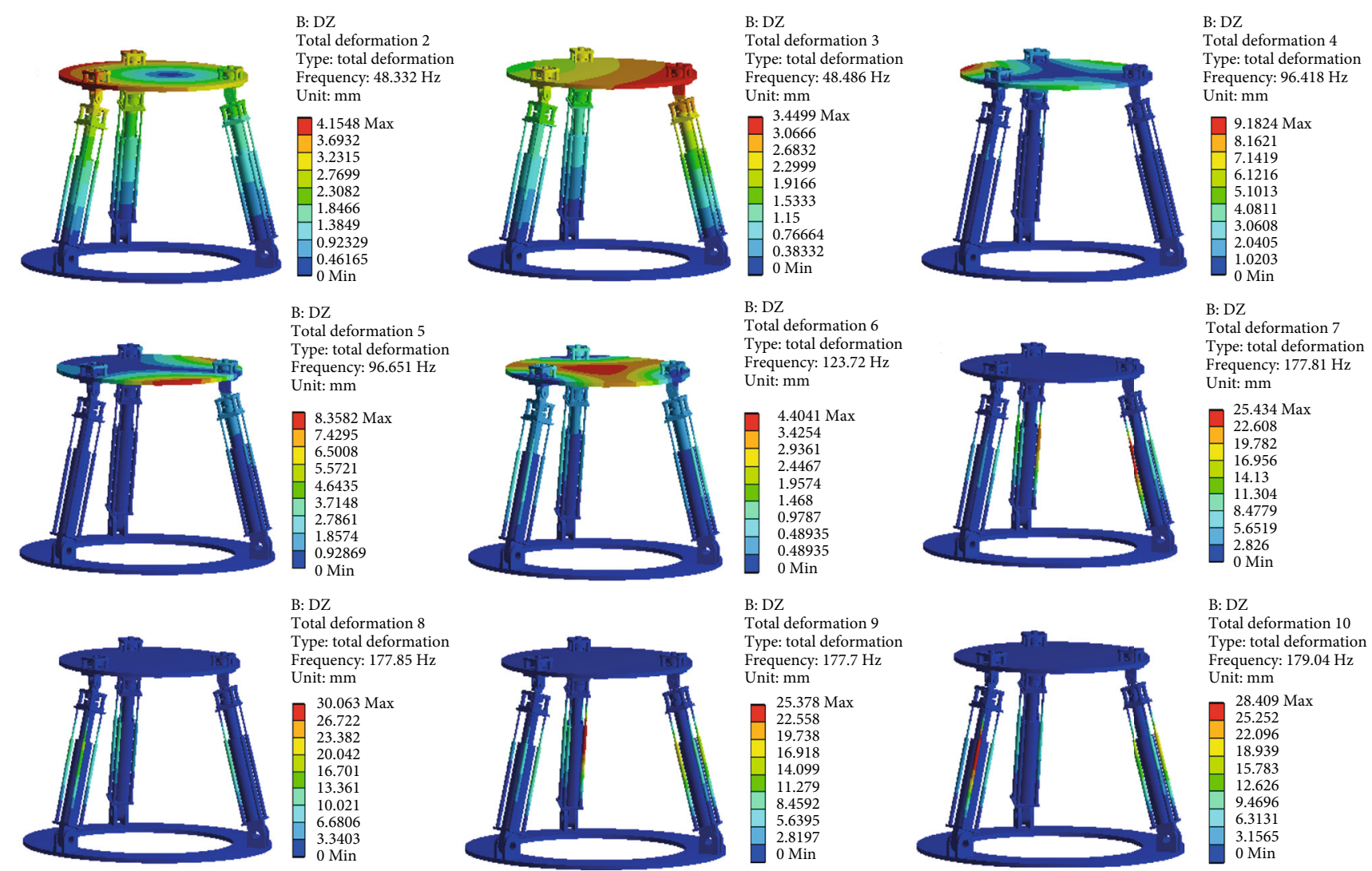

FIGURE 21: Vibration cloud figures of adjusting platform.

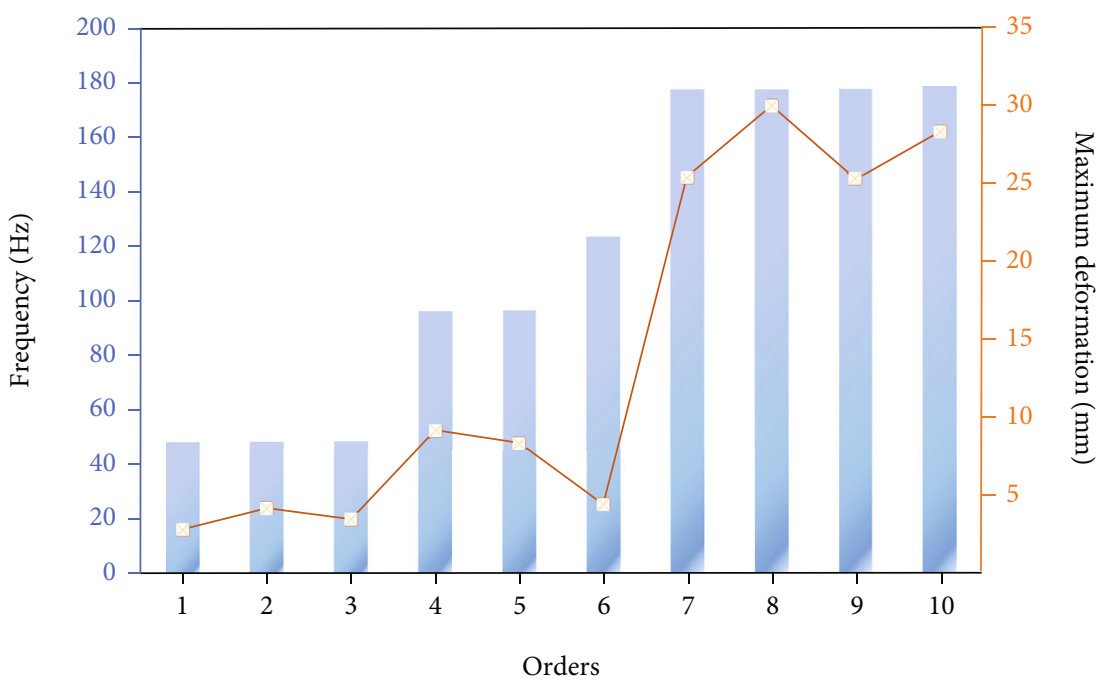

FIGURE 22: Natural frequencies and the biggest deformation of adjusting platform.

deformation mainly concentrated on the side slender rod of the levelling guide linkage.

The corresponding natural frequencies of 10 orders and the maximum deformation of vibration modes at corresponding frequencies are shown in Figure 22. The maximum deformation of vibration mode at the 1, 2, 3, and 6 orders is $4.40 \mathrm{~mm}$. The maximum deformation of vibration mode occurs at the edge of the upper platform under the 4- and 5 -order natural frequencies, and most of the middle is almost invisible. Although the maximum deformation of the 7-10-order natural frequency vibration diagram is relatively large, it can be found that the upper levelling platform has no vibration deformation combined with the Figure 21. On the whole, the vibration mode of the platform is relatively stable within the natural frequency range. Combined with the normal working environment of the adjusting platform, the vibration mode occurring under the 10-order natural frequencies of the platform will not affect its work, and 


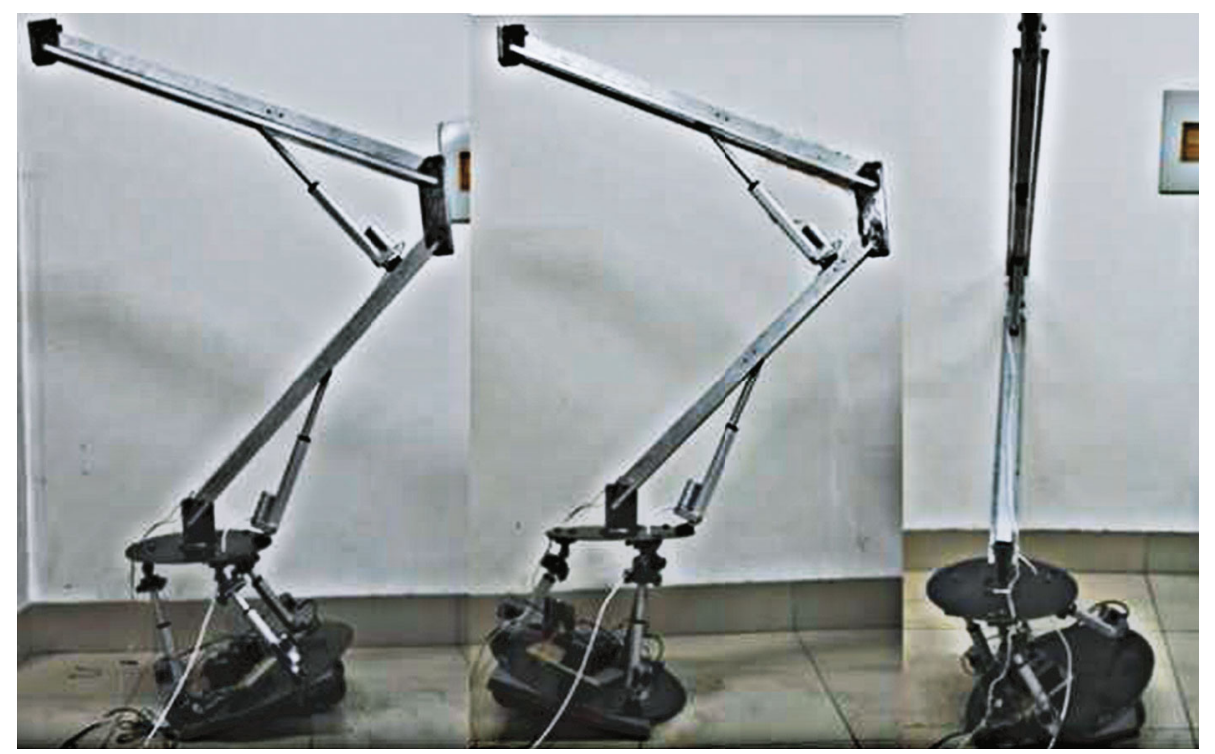

Figure 23: Adjusting platform state diagrams of different situations.

there is no need to remove these natural frequencies during normal operation.

4.3. Experimental Verification of Performance. The manufactured levelling platform controlled the movement of electric push rod using Arduino system. The functional test experiments shown in Figure 23 were carried out. The electric push rod was controlled movement under the slope inclined by $20^{\circ}$. Levelling was obtained in different situations. The levelling platform achieved the desired effect.

\section{Conclusions}

This paper studied the type synthesis theory of multilinkage robot and proposed the modular combination type synthesis method with virtual center of rotation. In order to verify the feasibility of the method, the aerial working platform and selfadaptive levelling platform were designed and manufactured based on the proposed method. The designed aerial working platform added virtual center of rotation achieved $360^{\circ}$ rotating large workspace, more compact mechanical structure, and higher productivity than the scissor-type and mast-type aerial working platforms. The designed adaptive levelling platform was tested at different inclinations. It could ensure the levelling of the upper surface. The study enriched and improved the theory of construction machinery synthesis. It provided a new idea for configuration design of multilinkage robot.

\section{Data Availability}

No data were used to support this study.

\section{Conflicts of Interest}

The authors declare that there is no conflict of interest regarding the publication of this paper.

\section{Acknowledgments}

This research was funded by the National Natural Science Foundation of China (Grant No. 52075306).

\section{References}

[1] T. L. Yang, H. P. Shen, and A. X. Liu, "Thrust Prediction of Underwater Blade-propeller-type Thrusters under Quasi-cavitation," Journal of Mechanical Engineering, vol. 56, no. 17, pp. 1-15, 2020.

[2] J. J. Yu, K. Liu, and X. W. Kong, "State of the art of multi-mode mechanisms," Journal of Mechanical Engineering, vol. 56, no. 19, pp. 14-27, 2020.

[3] N. L. Chau, N. T. Tran, and T. P. Dao, "An optimal design method for compliant mechanisms," Mathematical Problems in Engineering, vol. 2021, Article ID 5599624, 18 pages, 2021.

[4] H. Li and D. G. Huang, "Aerodynamic optimization design of a multistage centrifugal steam turbine and its off-design performance Analysis," International Journal of Rotating Machinery, vol. 2017, Article ID 4690590, 2017.

[5] G. L. Jia, B. Li, H. L. Huang, and D. Zhang, "Type synthesis of metamorphic mechanisms with scissor-like linkage based on different kinds of connecting pairs," Mechanism and Machine Theory, vol. 151, p. 103848, 2020.

[6] G. L. Jia, H. L. Huang, S. Wang, and B. Li, "Type synthesis of plane-symmetric deployable grasping parallel mechanisms using constraint force parallelogram law," Mechanism and Machine Theory, vol. 161, p. 104330, 2021.

[7] S. Wang, Y. Li, and Z. Chen, "Type synthesis of rehabilitation mechanism of variable axis bio-fusion knee joint," Journal of Mechanical Engineering, vol. 56, no. 11, pp. 72-79, 2020.

[8] C. Zhang, X. Liu, and C. Ni, "Type synthesis of mobile mechanisms with multiple modes based on single-loop kinematic chains," Journal of Mechanical Transmission, vol. 44, no. 4, pp. 17-25, 2020.

[9] Y. Q. Li, Y. Zhang, and Y. Guo, "New method for type synthesis of 2R1T redundant driven parallel mechanisms," Journal of Mechanical Engineering, vol. 55, no. 23, pp. 25-37, 2019. 
[10] Y. Lu, Y. Yang, and L. Jie, "Type synthesis of 5-DoF parallel mechanisms with different submechanisms," Mathematical Problems in Engineering, vol. 2018, Article ID 6021702, 13 pages, 2018.

[11] L. Li, Y. Fang, S. Guo, H. Qu, and L. Wang, "Type synthesis of a class of novel 3-DOF single-loop parallel leg mechanisms for walking robots," Mechanism and Machine Theory, vol. 145, p. 103695, 2020.

[12] Y. B. Zhang, K. M. Wang, and F. L. Lu, "Type synthesis of uncoupled two-rotational parallel mechanism with one pure constraint branch," China Mechanical Engineering, 2021.

[13] X. D. Meng, F. Gao, S. F. Wu, and Q. J. Ge, "Type synthesis of parallel robotic mechanisms: Framework and brief review," Mechanism and Machine Theory, vol. 78, pp. 177-186, 2014.

[14] L. Li, Y. Fang, and L. Wang, "Type synthesis of single-loop 3T1R-parallel mechanisms with a multi-DOF drive system," Mechanism and Machine Theory, vol. 163, p. 104373, 2021.

[15] Z. G. Gao, L. B. Zeng, B. He, T. Luo, and P. Zhang, "Type synthesis of non-holonomic spherical constraint underactuated parallel robotics," Acta Astronautia, vol. 152, pp. 509-520, 2018.

[16] T. Sun and X. M. Huo, "Type synthesis of 1T2R parallel mechanisms with parasitic motions," Mechanism and Machine Theory, vol. 128, pp. 412-428, 2018.

[17] Y. M. Song, P. P. Han, and P. F. Wang, "Type synthesis of $1 \mathrm{~T} 2 \mathrm{R}$ and 2R1T parallel mechanisms employing conformal geometric algebra," Mechanism and Machine Theory, vol. 121, pp. 475-486, 2018.

[18] F. G. Xie, X. J. Liu, Z. You, and J. Wang, "Type synthesis of 2T1R-type parallel kinematic mechanisms and the application in manufacturing," Robotics and Computer-Integrated Manufacturing, vol. 30, no. 1, pp. 1-10, 2014.

[19] G. L. Chen, J. Wang, H. Wang, C. Chen, V. Parenti-Castelli, and J. Angeles, "Design and validation of a spatial two-limb 3R1T parallel manipulator with remote center-of-motion," Mechanism and Machine Theory, vol. 149, p. 103807, 2020.

[20] W. Ye, B. Zhang, and Q. C. Li, "Design of a 1R1T planar mechanism with remote center of motion," Mechanism and Machine Theory, vol. 149, p. 103845, 2020.

[21] K. Y. Wu, F. Zhang, G. H. Cui, J. Sun, and M. Zheng, "Kinematics and force transmission analysis of a decoupled remote center of motion mechanism based on intersecting planes," Journal of Mechanisms and Robotics, vol. 13, no. 2, 2021.

[22] A. H. Khan, S. Li, and X. W. Cao, "Tracking control of redundant manipulator under active remote center-of-motion constraints: an RNN-based metaheuristic approach," Science China Information Sciences, vol. 64, no. 3, 2021.

[23] Y. Yang, H. Liu, H. Y. Zheng, Y. Peng, and Y. Yu, “Two types of remote-center-of-motion deployable manipulators with dual scissor-like mechanisms," Mechanism and Machine Theory, vol. 160, p. 104274, 2021.

[24] F. G. Wang, Y. L. Shi, and J. Zhang, "Dynamics modeling and simulation of prostate needle-insertion robot," Journal of Mechanical \& Automation, vol. 36, no. 12, pp. 1314-1319, 2019.

[25] Y. N. Chang, Z. S. Wang, and H. Wang, "Research on modeling of elastic element in RCC," Aerospace Shanghai, vol. 33, no. 5, pp. 57-62, 2016.

[26] J. Liu, X. H. Tao, and Y. G. Li, "Application summary of remote center compliance wrist," Development \& Innovation of Machinery \& Electrical Products, vol. 24, no. 2, pp. 74-76, 2011.
[27] J. H. Liu, S. S. Bi, and M. L. Sun, "Optimal design for passive robot in rough terrain based on VCM," Journal of Beijing University of Aeronautics Astronautics, vol. 35, no. 2, pp. 261-265, 2009.

[28] T. L. Yang, A. X. Liu, and Y. F. Luo, Theory and Application of Robot Mechanism Topology, Science Press, Beijing, 2012.

[29] X. Liu, B. Zhong, and Y. H. Chu, "Design and analysis of lifting and leveling mechanism for new orchard picking platform," Journal of Chinese Agricultural Mechanization, vol. 41, no. 2, pp. 80-85,106, 2020.

[30] L. Stawinski, J. Zaczynski, A. Morawiec, J. Skowronska, and A. Kosucki, "Energy consumption structure and its improvement of low-lifting capacity scissor lift," Energies, vol. 14, no. 5, p. 1366, 2021

[31] Y. Xiong, Y. Ge, L. Grimstad, and P. J. From, “An autonomous strawberry-harvesting robot: Design, development, integration, and field evaluation," Journal of Field Robotics, vol. 37, no. 2, pp. 202-224, 2020.

[32] H. Zhao, "Design of a portable high-altitude hemispherical wide-range plucking device," Agricultural Science\&Technology and Equipment, vol. 283, no. 1, pp. 18-20, 2018.

[33] Y. Xiao, Y. Fu, and T. Liu, "Design and experimental study of the portable motor-driven walnut picking machine," Journal of Agricultural Mechanization Research, vol. 40, no. 1, pp. 57-62, 2018.

[34] J. Cai, C. Yu, and T. Gao, "Designation on fully automatic high-altitude fruit picker," JOURNAI OF DALIAN UNIVERSITY, vol. 38, no. 6, pp. 12-13, 2017.

[35] G. Fan, Z. Li, and W. Mao, "Structure parameter optimization and experiment of orchard platform based on workspace," Transactions of the Chinese Society for Agricultural Machinery, vol. 52, no. 4, pp. 34-42, 2021.

[36] J. T. Ma, Y. Wang, and Y. He, "Motion planning of citrus harvesting manipulator based on informed guidance point of configuration space," Transactions of the CSAE, vol. 35, no. 8, pp. 100-108, 2019.

[37] Y. J. Zheng, S. J. Jiang, and B. T. Chen, "Review on technology and equipment of mechanization in hilly orchard," Transactions of the Chinese Society for Agricultural Machinery, vol. 51, no. 11, pp. 1-20, 2020.

[38] G. Y. Cai, H. H. Weng, and H. Y. Zhou, "Design of fruit picking robot for high-altitude operation," Light Industry Machinery, vol. 34, no. 1, 2016.

[39] J. Barnett, M. Duke, C. K. Au, and S. H. Lim, "Work distribution of multiple Cartesian robot arms for kiwifruit harvesting," Computers and Electronics in Agriculture, vol. 169, article 105202, 2020.

[40] X. Z. Ren, F. M. Li, and Y. J. Wei, "Finite element simulation design and high altitude picking test of intelligent mango vehicle," Journal of Agricultural Mechanization Research, vol. 12, pp. 91-95, 2020.

[41] X. Chen, Z. Chen, and J. Liu, "Development of automated mast hoisting equipment," in In the proceedings of the 30th Chinese Control and Decision Conference, CCDC, pp. 4383-4387, 2018.

[42] H. Su, Research on Key Technology of Hybraulic Handing Robot Motion Control System, Shandong University of Technology, 2019. 